\title{
Scaling relations and finite-size scaling in gravitationally correlated lattice percolation models
}

\author{
Chen-Ping Zhu ${ }^{1} *$ Long-Tao Jia ${ }^{1}$, Long-Long Sun ${ }^{1}$, Beom Jun \\ $\mathrm{Kim}^{2}$, Bing-Hong Wang ${ }^{3}$, Chin-Kun $\mathrm{Hu}^{4,5}+\dagger^{\dagger}$ and H. E. Stanley ${ }^{6}$ \\ ${ }^{1}$ College of Science, Nanjing University of \\ Aeronautics and Astronautics, Nanjing 210016, China \\ ${ }^{2}$ BK21 Physics Research Division and Department of Physics, \\ Sungkyunkwan University,Suwon 440-746, Korea \\ ${ }^{3}$ Department of Modern Physics, University of \\ Science and Technology of China, 200093, China \\ ${ }^{4}$ Institute of Physics of Academia Sinica, Taipei 11529, Taiwan \\ ${ }^{5}$ Department of Physics, National Dong Hwa University, Hualian 97401, Taiwan and \\ ${ }^{6}$ Center for Polymer Studies, Department of Physics, \\ Boston University, Boston, Massachusetts 02215, USA
}

(Dated: December 30, 2019) 


\begin{abstract}
In some systems, the connecting probability (and thus the percolation process) between two sites depends on the geometric distance between them. To understand such process, we propose gravitationally correlated percolation models for link-adding networks on the two-dimensional lattice $G$ with two strategies $S_{\max }$ and $S_{\min }$, to add a link $l_{i, j}$ to connect site $i$ and site $j$ with mass $m_{i}$ and $m_{j}$, respectively; $m_{i}$ and $m_{j}$ are sizes of the clusters which contain site $i$ and site $j$, respectively. The probability to add the link $l_{i, j}$ is related to the generalized gravity $g_{i j} \equiv m_{i} m_{j} / r_{i j}^{d}$, where $r_{i j}$ is the geometric distance between $i$ and $j$, and $d$ is an adjustable decaying exponent. In the beginning of the simulation, all sites of $G$ are occupied and there is no link. In the simulation process, two inter-cluster links $l_{i, j}$ and $l_{k, n}$ are randomly chosen and the generalized gravities $g_{i j}$ and $g_{k n}$ are computed. In the strategy $S_{\max }$, the link with larger generalized gravity is added. In the strategy $S_{\min }$, the link with smaller generalized gravity is added, which include percolation on the Erdős-Rényi random graph and the Achlioptas process of explosive percolation as the limiting cases, $d \rightarrow \infty$ and $d \rightarrow 0$, respectively. Adjustable strategies facilitate or inhibit the network percolation in a generic view. We calculate percolation thresholds $T_{c}$ and critical exponents $\beta$ by numerical simulations. We also obtain various finite-size scaling functions for the node fractions in percolating clusters or arrival of saturation length with different intervening strategies.
\end{abstract}

PACS numbers: 89.75.Hc, 05.45.Df

*Electronic address: oldpigman1234@126.com

$\dagger$ Electronic address: huck@phys.sinica.edu.tw 


\section{INTRODUCTION}

The study of phase transitions and critical phenomena has attracted much attention in recent decades [1 5]. The key concepts in such studies include critical point, critical exponent, universality, scaling, and finite-size scaling function [6-11]. In this paper, we will address the problem of critical behavior of network percolation.

Network percolation has been playing an important role as a simplified model to understand spreading processes of message, disease, matter and dynamic processes in complex systems [12 20]. It has been attracting more and more attention from physics and other research communities. With the paradigm of complex networks, nodes representing individuals and links interactions among them, percolation in networks serves as a bridge connecting classical model of statistical physics and practical problems in various fields [21]. However, further application of the theory is somewhat limited since links in networks are often in the sense of topology, i.e., connecting relations without taking into account the geometric distance. By contrast, it is necessary to have a geometric controllability in network percolation, i.e., to facilitate or inhibit network percolation in link-adding processes based on the geometric distance, which motivates us to free ourselves from the constraint of purely topological connection between nodes in previous models. As the consequence, intervening strategies for this kind of correlated percolation [22 25] lead to new scaling relations and finite-size scaling.

In some systems, the connecting probability (and thus the percolation process) between two sites depends on the geometric distance between them. Mobile ad hoc network (MANET) [26], as an example, is a new wireless communication system for temporal assembly of moving members. The flooding mechanism [26] of its message pervading can be viewed as a percolation process. A MANET should assign proper transmission range [26-28] for all nodes to prevent interference among themselves, and to save energy for longer lifetime of the network since they could not be recharged during motion. Therefore, direct communications can happen only inside speaking nodes' transmission circles [28, 29], outside which nodes are linked in a manner of multi-hop (indirect wireless connections through successive relays).

Here, global connectivity [27] relies on a suitable design of transmission range adapting to the occupation density of nodes on a two-dimensional (2D) plane. Besides, the traffic flux and bilateral trade volumes between two cities or countries are found to be proportional to 
the gross economic quantity of each side, and inversely proportional to the distance between them. Therefore, gravitation models [30, 31] are often used to understand empirical data in various situations. The spread of the ground traffic congestion could be viewed as another kind of distance-related percolation in which Manhattan distance (the summation over projected lengths of geometric distance along two perpendicular directions) plays a key role. Therefore, Li, et al. [32] pointed out that a power-law distance-decaying link-adding probability in a 2D lattice could optimize ground traffic under certain constraints on total cost. Moreover, a disaster gravity mobility model [33] for MANET defines a maximum distance at which an event affects objects in a gravitational style. That is why pervasive disasters or rush-hour congestion can cause percolation-like phenomena between objects [34]. In short, to properly understand percolation in some real networks, we should not ignore linking effect related to the geometric distance.

In practice, people often need to combine percolation process with strategies to achieve better results of coevolutionary processes. In the situation of massive disaster, base stations of mobile communication often suffer from black-out, yielding a large scale of disconnected population. In order to deliver messages, energy, and matter supplies in disaster relief efforts to all panicked people as soon as possible, one needs to facilitate percolation in link-adding networks of vehicles equipped with MANET nodes or other systems. While in other situations, such as the spread of ground traffic congestion and epidemics [28, 35] which depend on the geometric distance, one should design effective measures to inhibit percolation. One possible algorithm for such inhibition is the product rule (PR) proposed by Achlioptas, et al. [21] and other models suggested afterwards [36 55]. Original PR starts from a network with isolated nodes as the initial condition. During the evolution process, a node $i$ is labeled by its mass (or called size) $m_{i}$ which is the number of connected nodes in the cluster that includes node $i$. Two topological links are randomly put into the set of the nodes at every time step, and only the one connecting two nodes $i$ and $j$ with smaller product of masses $\left(m_{i} m_{j}\right)$ is retained. This rule postpones the development of the giant component, and a sharp change of the fraction of the nodes in the largest cluster is observed, which has been called "explosive percolation". Instead of investigating the nature of such an unusual continuous [56 59] or discontinuous [36, 37] phase transition, we are concerned with how to facilitate or inhibit percolation in a kind of extended scheme in network growth process.

In this paper, we propose several percolation schemes on a $2 \mathrm{D}$ plane with link-adding 
rules depending on the geometric distance, which takes the form similar to Newton's gravity. Simply by adopting the strategy of either maximum or minimum gravity in successive linking steps for different cases, one can facilitate or inhibit network percolation in a systematic way. The observed size of the largest component (cluster), and average connection lengths of various link types, are revealed to follow scaling relations which were not recognized in purely topological percolation models. The present scheme gives a generic picture for percolation processes in real systems which are often inevitably geometrically constrained.

\section{MODELS}

Suppose $N$ isolated nodes are uniformly scattered on a two-dimensional (2 D) plane with the edge length $L$, hence $N=L^{2}$. For the convenience to calculate distance, the plane is discretized with a triangular lattice $G$. Each vertex of the triangles is occupied by a node.

As in product rule (PR) of Achlioptas process [21], we pick randomly two pairs $[(i, j)$ and $(k, l)$ ] of nodes in the plane at every time step. For the pair $(i, j)$ (and for $(k, l)$ likewise), we compute the generalized gravity defined by $g_{i j} \equiv m_{i} m_{j} / r_{i j}^{d}$, where $m_{i}$ and $m_{j}$ are the number of sites of the clusters which include site $i$ and site $j$, respectively, $r_{i j}$ is the geometric distance between $i$ and $j$, and $d$ is an adjustable decaying exponent. Once we have $g_{i j}$ and $g_{k l}$, we have two choices in selecting which link should be retained. For the case of the maximum gravity strategy (we call it $S_{\max }$ ) we connect the pair with the larger value of the gravity, e.g., the link $(i, j)$ is made if $g_{i j}>g_{k l}$, and the link $(k, l)$ otherwise. We also use the minimum gravity strategy $\left(S_{\min }\right)$ in which we favor the pair of nodes with smaller gravity to make connection. The two strategies, $S_{\max }$ and $S_{\min }$, lead the link-adding networks to evolve along the opposite percolation processes. Generally speaking, $S_{\max }$ facilitates the percolation process, whereas $S_{\min }$ inhibits it similar to explosive percolation [21, 36, 56 58]. All such generalized gravity values are calculated inside the circular transmission range with the radius $R$ centered at one of nodes $i$ and $j$ as the speaking node [28, 29] in a MANET, for example.

For the different limits of parameters $R$ and $d$, we have two cases. Case 1: With the transmission range $R \rightarrow \infty$, we have a generalized gravitation rule which is an extension [33] of widely used gravitation model $(d=1)[30,31]$ with the decaying exponent $d$ tunable. Case

2: With both adjustable values of radius $R$ and exponent $d$, we have the gravity rule [30, 31] 
inside the transmission range. It can describe the communication or traffics with constrained power or resources.

\section{SIMULATION RESULTS}

All simulations are carried out on the $L \times L$ triangular lattice of the size $N=L \times L$ with $L=32,64,128$ and 256, respectively. We simulate either of strategy $S_{\max }$ or $S_{\min }$. The total number of links equating to that of time-steps is divided by $N$, which is defined as $T$. The mass of the largest component divided by $N$ makes up the observable $C_{1}$, the node fraction of the largest component. The algorithm in the present model is similar to that of Ref. [39] including the rule of intra-cluster priority, except distance-decaying exponent $d$ and transmission radius $R$ used. And similar time-dependent variation of fractions of different types (I: both inter-clusters; II: one inter-cluster and the other intra-cluster; and III: both intra-clusters, see Fig. 2 in [39]) of links retained [39, 55] are also observed near the threshold of percolation. All results presented in this work are obtained from the average over 100 different realizations of network configurations.

For strategy $S_{\max }$ in Case 1, the percolation threshold decreases from the limit $T_{c}=0.5$ for the Erdős-Rényi (ER) random graph [see Fig. 1(a)]. As the exponent $d$ decreases, $T_{c}$ shifts downward (e.g., $T_{c}=0.37$ for $d=0.2$ and $T_{c}=0.36$ for $d=0.01$ ). Following the standard manipulation [60], we obtain a group of decaying - exponent $d$ - dependent percolation thresholds $T_{c}$, and corresponding critical exponents $\beta_{1}$ in the probability for a node to be in the percolating cluster:

$$
C_{1} \sim\left(T-T_{c}\right)^{\beta_{1}} \text { for } T \rightarrow T_{c}+
$$

Numerical results for $S_{\max }$ in Case 1 are listed in Table I. Obviously, $T_{c}$ and $\beta_{1}$ increase with d. Figure 1(b) shows that for $S_{\min }, T_{c}$ and the exponent $\beta_{1}$ also depends on $d$.

In addition, another special point $T_{0}$ attracts our attention. Curves $C_{1}(T)$ of Fig. 1(a) for different $d$ cross approximately at a point $T_{0}\left(>T_{c}\right)$. Let $t=\left(T-T_{0}\right) / T_{0}$, then, $C_{1}(T)$ can be roughly re-scaled as

$$
C_{1} \sim d^{-\omega} f\left(t d^{\epsilon}\right)
$$

for different exponents $0.2<d \leq 2$ (inset of Fig. 1(a), except the situation $d=0.2$ with a dashed green line), where $T_{0}=0.78, \omega=0.01, \epsilon=0.20$, and $f(x)$ is a universal scaling 
function, which is similar to the super-scaling behavior studied by Watanabe and $\mathrm{Hu}$ [61].

With the strategy $S_{\min }$ in Case 1, PR [21] can be resumed by letting $d \rightarrow 0$, with the threshold $T_{c}$ approaching 0.888 which is the transition point of Achlioptas-type percolation [21]. On the other hand, $d \rightarrow \infty$, the gravity values for both candidate links become indistinguishable and thus any one of the two is selected arbitrarily, which resumes the case of percolation in growing ER random graph. Fig. 1 (b) illustrates these two limiting cases and intermediary ones between them with $L=128$.

According to the priority rules distinguishing candidate links into three types as shown in Figure 2 of [39], we calculated the average lengths $l_{I}, l_{I I}$ and $l_{I I I}$ of type-I, type-II and type-III links, respectively, as the summations of specific link-lengths over corresponding numbers of such types of links. The finally saturated average lengths of both type-II and type-III links are $l_{0}=131.9$ for $L=128$ (see Fig.2). Such saturated value is reached for $T \geq T_{s}=1.0$.

To find the average length $l$ till time step $T$ with strategy $S_{\max }$ in case 1 , we do ensemble average on geometric lengths of retained links under different exponents $(d=$ $0.2,0.5,1.0,2.0,3.0$ and 5.0 ) for the lattice with the edge length $L=128$. Simulation results for three types of links [39, 55] are shown in Fig. 2. Temporal variations of normalized average lengths of type-III links [39, 55] are re-scaled to collapse very well into a single curve as shown in Fig. 3. Therefore, we get the following scaling behavior:

$$
l / l_{0} \sim d^{-\lambda} F\left(d^{T} T\right)
$$

where $l_{0}=131.90$ (see Fig. 2), $\lambda=-0.001, \tau=0.005$ and $F(x)$ is a universal scaling function.

As seen in Fig.2, averaged lengthes $l$ of both type-II and type-III links grow monotonically until they get saturated. Actually, they approach the saturated average length $l_{0}=131.9$ (see Fig. 2) in $d$-dependent paces. Average length $l$ in any growth step (T) for a smaller $d$ is longer than those with larger $d$, because strategy $S_{\max }$ favors the former links, and the links with a larger $d$ starts to be realized later on average than those with smaller $d$ due to the same reason. Interestingly, $d$-dependent average lengths for each type of links have their own universal scaling functions, which are illustrated in Fig. 4 and Fig. 5, respectively. The scaling behavior for type-II links in case 1 to arrive at saturated average length $l_{0}$ reads: 


$$
p_{2} \sim g\left((T-1.0)^{\alpha_{2}} d^{\gamma_{2}}\right)
$$

where $\alpha_{2}=-0.35$ and $\gamma_{2}=-0.03$, respectively and $g(x)$ is a universal scaling function valid for $0.2 \leq d \leq 5.0$. Meanwhile, the scaling behavior for type-III links in case 1 to arrive at saturated average length $l_{0}$ reads:

$$
p_{3} \sim S\left((T-1.0)^{\alpha_{3}} d^{\gamma_{3}}\right)
$$

where $\alpha_{3}=-1.0$ and $\gamma_{3}=-0.08$, respectively and $S(x)$ is a universal scaling function valid for $0.2 \leq d \leq 5.0$.

In addition, the difference of average lengths between type-II and type-III links $\left(l_{I I}-l_{I I I}\right)$ is exactly coherent with the difference of average fractions between these two types of links $\left(F_{I I}-F_{I I I}\right)$ at the same $T$, which is shown in Fig. 6. Therefore, a universal function exists for $\left(l_{I I}-l_{I I I}\right)$ vs. $\left(F_{I I}-F_{I I I}\right)$ in the simulated range of $d(0.2 \leq d \leq 5.0)$. While pure Achlioptas process [21] does not share the same property (shown in blue line). Obviously, Fig.3 - Fig.6 and corresponding scaling relations (formulas (3), (4), and (5)) can not be accounted as trivial ones, since they only happen to the present schemes based on the classification in ref. [39, 55].

Simulations for Case 2 reveal combined effect of transmission range and gravitation. Following the standard manipulation in Ref. [60], we obtain a group of decaying - exponent $d$ - dependent percolation thresholds $T_{c}$, and corresponding critical exponents $\beta_{2}$ in the probability for a node to be in the percolating cluster, with the same form as formula (1) but different exponents $\beta_{2}$.

$$
C_{2} \sim\left(T-T_{c}\right)^{\beta_{2}} \text { for } T \rightarrow T_{c}+
$$

Numerical results for $S_{\min }$ in Case 2 are listed in Table II which shows that $T_{c}$ and $\beta_{2}$ depend on $d$.

In addition, another special point $T_{0}$ attracts our attention. Rough scaling relations with strategy $S_{\min }$ are obtained for a range of $R(3<R \leq 8)$ and distance-decaying exponent $d$ $(0.2<d<2.0)$ : 


$$
C_{2} \sim\left(d / d_{0}\right)^{-\theta} h\left[t\left(d / d_{0}\right)^{\phi}\right]
$$

for different $d$, where $T_{0}=1.0, \theta=0.005, \phi=-0.50, d_{0}=0.5$, and $h(x)$ is an approximate universal scaling function. For this scaling relation, the validation range of decaying exponent $d$ and transmission range $R$ need to adapt to each other, since the effect of a weak decay with a small exponent $d$ would be diminished by a small enough $R$ (e.g., we must have $d>0.5$ for $R=4$ ), and strong enough decay (large $d$ ) would ruin the effect of a large $R$ (e.g., we must have $d<5.0$ for $R=8$ ). A modest example for $R=5$ is shown in Fig. 7(a) (The scaling is roughly valid for $0.2<d<2.0$ ).

Besides, a rough scaling behavior with strategy $S_{\max }$ for different $R$ and $d$ reads:

$$
C_{2} \sim R^{-\delta} H\left(t \rho^{\eta}\right)
$$

for $R>3$, where $\rho=\left(R-R_{0}\right) / R_{0}, R_{0}=2, \eta=-0.10, \delta=-0.005, T_{0}=1.0$, and $H(x)$ is an approximate universal scaling function, which is shown in Fig. 7(b) (scaling is only valid for a small range $(4<R \leq 8)$. Here, $T_{0}$ is another special point where average lengths of type-II and type-III links arrive at the same level, and fractions of type I and III links [39, 55] get a balance, meanwhile the fraction of type II links arrives at its summit [62]. Also, suitable match between parameters $d$ and $R$ is required. Otherwise, this scaling behavior is invalid, just as the case $R=4$ in the inset of Fig. 7(b).

Inhibitory strategy $S_{\min }$ in Case 2 produces the largest threshold on a 2D plane to the best of our knowledge. Through finite-size transformation we check the critical point $T_{c}$. The scaling behaviors of node fraction $C_{2}$ and susceptibility $\chi$ defined as $\chi \equiv\left[\left\langle C_{2}^{2}\right\rangle-\left\langle C_{2}\right\rangle^{2}\right] / N \quad$ [40, 63] are

$$
\begin{gathered}
C_{2} \sim N^{-\beta / \nu} Q\left(\left(T-T_{c}\right) N^{1 / \nu}\right), \\
\chi \sim N^{\gamma / \nu} Z\left(\left(T-T_{c}\right) N^{1 / \nu}\right),
\end{gathered}
$$


where $1 / \nu=0.2, \beta / \nu=0.005, \gamma / \nu=0.995$, and $Q(x)$ and $Z(x)$ are universal scaling functions. Therefore, a scaling law of continuous phase transition

$$
\beta / \nu+\gamma / \nu=1
$$

remains valid for two scaling relations for different parameter sets $(R, d)$, which is verified well although scaling relations (7) is limited within a small range for $S_{\min }$ in Case 2. Similar scaling law has been obtained by Radicchi et al. [40] for scale-free networks but with different sets of exponents. Therefore, the present one in Fig. 8 should be concluded into a different universality class. Numerical evidence of $S_{\min }$ in Case 2 for $R=2, d=2.0$ with $L=$ 32, 64, 128 and 256 are shown as an example in Fig. 8(a) and Fig. 8(b), with the percolation threshold as large as $T_{c} \simeq 1.5$, as an example of $S_{\min }$ in Case 2. Insets of them illustrate the re-scaled results of $C_{2}$ and $\chi$ (see formulas (9) and (10)), respectively.

\section{DISCUSSION AND CONCLUSIONS}

It should be noted that scaling relations illustrated in Fig. 1, Fig. 3, Fig. 4, Fig. 5 and Fig. 7 (formulas (2), (3), (4), (5), (7) and (8)) are not referring to critical points $T_{c}$ of pertinent percolation in specific gravitational distance - decaying schemes. Instead, they are referring to kind of special points $T_{0}$ which are $d$ governing or $(d, R)$ coordinately controlled, and worthy of further investigation. Among them formula (1) around $T_{0}$ for $S_{\max }$ in case 1 is approximately valid. And formula (5) and (6) are valid only for properly matched sets of $R$ and $d$. By contrast, Fig. 8 and corresponding scaling relations, i.e. formulas (7), (8) and (9) referring to order parameter $C_{2}$ and $\chi$ around $T_{c}$ are quite solid.

The gravitationally correlated lattice percolation models (GCLPMs) introduced in this paper are new models of long-range correlated percolation [22 25], and they are in different universality class from the existing correlation percolation model, e.g. the scaling law mentioned in [22] is violated and the PR is merged into the bond-occupation schemes. From this viewpoint we can understand a different saturation effect of $S_{\max }$ for decaying exponent $d \geq 3.0$ in Fig. 1(b) and limited validation ranges of $d$ for all scaling relations relevant to correlations in Case 2 with strategy $S_{\min }$. 
Intervening schemes in the present gravitational correlated percolation have predicted rich scaling relations. With the link-adding network schemes depending on gravitational distance-decaying strategies $S_{\max }$ or $S_{\min }$, we designed different ways to facilitate or inhibit network percolation on the $2 \mathrm{D}$ plane from a generic view of continuous phase transition. The adjustable transition threshold covers the range from 0.36 to 1.5 with the present simulations, which provides an approach to tuning critical point $T_{c}$ precisely according to requirement of different systems. Moreover, the approaches to re-scale time (the number of edges $T$ ) of a growing network with distance information would reveal more critical spatiotemporal properties of co-evolutionary processes. They could get broader applications than previous network percolation models constrained in topological sense when parameters $d$ and $R$ are properly selected for practical problems.

The GCLPMs introduced in this paper can inspire many interesting problems for further studies. In the present paper, we only simulate the GCLPM on the plane triangular (pt) lattice and obtain the finite-size scaling function only for the pt lattice. It has been found that bond and site percolation models on the square (sq), plane triangular (pt), and honeycomb (hc) lattices can have universal finite-size scaling functions when the aspect ratios of the sq, pt, and hc lattices are chosen to have the relative sizes 1: $\sqrt{3} / 2: \sqrt{3}[8,9$, 64]. An argument about why to choose such aspect ratios can be found in the Appendix C of [2]. We can simulate the GCLPM on the sq, pt and he lattices whose aspect ratios have the relative sizes 1: $\sqrt{3} / 2: \sqrt{3}$ to obtain the universal finite-size scaling functions of the GCLPM on the sq, pt and he lattices.

The Ising model and the Potts model are important lattice models [1, 2, 65 68]. It has been found that the Ising model on the sq, pt and he lattices whose aspect ratios have the relative sizes 1: $\sqrt{3} / 2: \sqrt{3}$ can have universal finite-size scaling functions [11, 69, 70]. It has been shown that the Ising model and the Potts model are corresponding to the 2-state and the $q$-state bond-correlated percolation models (qBCPM) [2, 63, 71], respectively. The 2-state bond correlated percolation model (2BCPM) is a special case of the qBCPM when $q=2$. The random bond percolation model is a special case of the qBCPM when $q=1$ [66]. To simulate the qBCPM, Swendsen-Wang has proposed a Swendsen-Wang algorithm [72], which can overcome the critical slowing down. $\mathrm{Hu}$ and Mak had used this algorithm to simulate the qBCPM on the sq and the simple cubic lattices [73]. Chen, Hu and Mak had developed a FORTRAN program to simulate the qBCPM on D-dimensional hypercubic 
lattices [74] based on the Swendsen-Wang algorithm [72]. In this paper, we modify the bond random percolation model to introduce the GCLPM. In the future, we can modify the qBCPM to include the concepts from the GCLPM. Such a model can be denoted as qBCPMGCLPM. We can simulate the qBCPM-GCLPM on the sq, pt and he lattices whose aspect ratios have the relative sizes $1: \sqrt{3} / 2: \sqrt{3}$ to find the universal finite-size scalings for the qBCPM-GCLPM. We can also study whether and how the qBCPM-GCLPM can show a first-order phase transition as parameters of the model, e.g. $q$ and $d$, are changed.

In summary, the GCLPM introduced in this paper can inspire many interesting problems for further studies.

\section{Acknowledgments}

C.P.Z. thanks H. Park, P. Holm, X.-S. Chen, M.-X. Liu and Z.-M. Gu for useful discussion. C. K. H. is indebted to R. M. Ziff for a critical reading of the manuscript. C.P.Z., L.T.J. and L.L.S. acknowledge financial support from National Natural Science Foundation of China (NNSFC) under Grants No. 11175086, 10775071 and 11775111. B.J.K. acknowledges the support from the National Research Foundation of Korea (NRF) grant funded by the Korea Government (MSIT) Grant No. 2017R1A2B2005957. C.K.H. is supported by Grant MOST 108-2112-M-259 -008.

[1] H. E. Stanley, Introduction to Phase Transitions and Critical Phenomena (Oxford Univ. Press, New York, 1971).

[2] C.-K. Hu, Historical Review on Analytic, Monte Carlo, and Renormalization Group Approaches to Critical Phenomena of Some Lattice Models, Chinese J. Phys. 52 (2014) 1-76. DOI: $10.6122 /$ CJP.52.1.

[3] E. Albayrak, The random distribution of the coordination numbers in the mixed spin- $1 / 2$ and spin-2 Blume-Capel model, Chinese J. Phys. 56 (2018) 2291-2296.

[4] C. N. Chen, C.-K. Hu, N. S. Izmailian, M. C. Wu, Specific heat and partition function zeros for the dimer model on the checkerboard B lattice: Finite-size effects, Phys. Rev. E 99 (2019) 012102 . 
[5] A. Feraoun, S. Amraoui, M. Kerouad, Critical and compensation behaviors of a mixed spin (5/2,2) Ising system on a graphene layer, Chinese J. Phys. 58 (2019) 98-108.

[6] V. Privman, M. E. Fisher, Universal critical amplitudes in finite-size scaling, Phys. Rev. B 30 (1984) 322-327.

[7] C.-K. Hu, Boundary conditions and scaling functions of percolation models, J. Phys. A 27 (1994) L813-L820.

[8] C.-K. Hu, C.-Y. Lin, J.-A. Chen, Universal Scaling Functions in Critical Phenomena, Phys. Rev. Lett. 75 (1995) 193-196; Erratum Phys. Rev. Lett. 75, 2786 (1995).

[9] C.-K. Hu, C.-Y. Lin, Universal scaling functions for numbers of percolating clusters on planar lattices, Phys. Rev. Lett. 77 (1996) 8-11.

[10] C.-Y. Lin, C.-K. Hu, Universal finite-size scaling functions for percolation on three-dimensional lattices, Phys. Rev. E 58 (1998) 1521-1527.

[11] Y. Tomita, Y. Okabe, C.-K. Hu, Cluster analysis and finite-size scaling for Ising spin systems, Phys. Rev. E 60 (1999) 2716-2720.

[12] C. Moore, M. E. J. Newman, Epidemics and percolation in small-world networks, Phys. Rev. E $61(2000) 5678$.

[13] D. S. Callaway, M. E. J. Newman, S. H. Strogatz, D. J. Watts, Network robustness and fragility: Percolation on random graphs, Phys. Rev. Lett. 85 (2000) 5468.

[14] S. Boccaletti, J. A. Almendral, S. Guan, I. Leyva, Z. Liu, I. Sendina-Nadal, Z. Wang, Y. Zou, Explosive transitions in complex networks' structure and dynamics: Percolation and synchronization, Phys. Rep.-Rev. Sec. Phys. Lett. 660 (2016) 1-94.

[15] X. Y. Zhang, Y. Zou, S. Boccaletti, Z. H. Liu, Explosive synchronization as a process of explosive percolation in dynamical phase space, Sci. Rep. 4 (2014) 5200.

[16] L. Hebert-Dufresne, A. Allard, Smeared phase transitions in percolation on real complex networks, Phys. Rev. Res. 1 (2019) 013009

[17] B. Karrer, M. E. J. Newman, L. Zdeborova, Percolation on Sparse Networks, Phys. Rev. Lett. 117 (2014) 208702.

[18] G. Bianconi, Rare events and discontinuous percolation transitions, Phys. Rev. E 97 (2018) 022314 .

[19] F. Radicchi, Predicting percolation thresholds in networks, Phys. Rev. E 91 (2015) 010801(R).

[20] A. Allard, L. Hebert-Dufresne, Percolation and the Effective Structure of Complex Networks, 
Phys. Rev. X 9 (2019) 011023.

[21] D. Achlioptas, R. M. D’Souza, J. Spencer, Explosive Percolation in Random Networks, Science 323 (2009) 1453.

[22] A. Weinrib, Long-range correlated percolation, Phys. Rev. B 29 (1984) 387.

[23] A. A. Saberi, Recent advances in percolation theory and its applications, Phys. Rep. 578 (2015) 1.

[24] J. Cheraghalizadeh, M. N. Najafi, H. Dashti-Naserabadi, H. Mohammadzadeh, Mapping of the Bak, Tang, and Wiesenfeld sandpile model on a two-dimensional Ising-correlated percolation lattice to the two-dimensional self-avoiding random walk, Phys. Rev. E 96 (2017) 052127.

[25] S. Zhang, L. Y. Zhang, M. Bouzid, D. Z. Rocklin, E. Del Gado, X.M. Mao, Correlated Rigidity Percolation and Colloidal Gels, Phys. Rev. Lett. 123 (2019) 058001.

[26] C. E. Perkins, Ad Hoc Networking. (Addison-Wesley, New York, 2000).

[27] L. Wang, C.-P. Zhu, Z.-M. Gu, Scaling of critical connectivity of mobile ad hoc networks, Phys. Rev. E 78 (2008) 066107.

[28] C.-P. Zhu, L Wang, X. T. Liu, Z. J. Yan, Directed Dynamic Small-World Network Model for Worm Epidemics in Mobile ad hoc Networks, Chin. Phys. Lett. 29 (2012) 010504.

[29] M. Nekovee, Worm epidemics in wireless ad hoc networks, New J. Phys. 9 (2007) 189.

[30] J. Tinbergen, Shaping the World Economy: Suggestions for an International Economic Policy (Twentieth Century Fund, New York, 1962).

[31] P. Poyhonen, A tentative model for the volume of trade between countries, Weltwirtschaftliches Archiv 90 (1963) 93-100.

[32] G. Li, S. D. S. Reis, A. A. Moreira, S. Havlin, H. E. Stanley, J. S., Jr. Andrade, Towards Design Principles for Optimal Transport Networks, Phys. Rev. Lett. 104 (2010) 018701.

[33] R. R. Roy, Handbook of Mobile Ad Hoc Networks for Mobility Models Part 2, pp. 443-482 (2011).

[34] D. Q. Li, B. W. Fu, Y. P. Wang, G. Q. Lu, Y. Berezin, H. E. Stanley, S. Havlin, Percolation transition in dynamical traffic network with evolving critical bottlenecks, Proc. Natl. Acad. Sci. U. S. A. 112 (2015) 669-672.

[35] C. P. Zhu, S. J. Xiong, Y. J. Tian, N. Li, K. S. Jiang, Scaling of directed dynamical small-world networks with random responses, Phys. Rev. Lett. 92 (2004) 218702.

[36] Y. S. Cho, S. Hwang, H. J. Herrmann, B. Kahng, Avoiding a Spanning Cluster in Percolation 
Models, Science, 339 (2013) 1185.

[37] S. Boettcher, V. Singh, R. M. Ziff, Ordinary percolation with discontinuous transitions, Nat. Commun. 3 (2012) 787.

[38] R. M. Ziff, Explosive Growth in Biased Dynamic Percolation on Two-Dimensional Regular Lattice Networks, Phys. Rev. Lett. 103 (2009) 045701.

[39] Y. S. Cho, J. S. Kim, J. Park, B. Kahng, D. Kim, Percolation Transitions in Scale-Free Networks under the Achlioptas Process, Phys. Rev. Lett. 103 (2009) 135702.

[40] F. Radicchi, S. Fortunato, Explosive Percolation in Scale-Free Networks, Phys. Rev. Lett. 103 (2009) 168701.

[41] N. A. M. Araújo, J. S. Andrade, Jr., R. M. Ziff, H. J. Herrmann, Tricritical Point in Explosive Percolation, Phys. Rev. Lett. 106 (2011) 095703.

[42] E. J. Friedman, A. S. Landsberg, Construction and Analysis of Random Networks with Explosive Percolation, Phys. Rev. Lett. 103 (2009) 255701.

[43] R. M. D’Souza and M. Mitzenmacher, Local Cluster Aggregation Models of Explosive Percolation, Phys. Rev. Lett. 104 (2010) 195702.

[44] N. A. M. Araújo, H. J. Herrmann, Explosive Percolation via Control of the Largest Cluster, Phys. Rev. Lett. 105 (2010) 035701.

[45] R. Cohen, D. J. Dawid, M. Kardar, Y. Bar-Yam, Unusual percolation in simple small-world networks, Phys. Rev. E 79 (2009) 066112.

[46] Y. S. Cho, S.-W. Kim, J. D. Noh, B. Kahng, D. Kim, Finite-size scaling theory for explosive percolation transitions, Phys. Rev. E 82 (2010) 042102.

[47] A. A. Moreira, E. A. Oliveira, S. D. S. Reis, H. J. Herrmann, J. S. Andrade, Jr., Hamiltonian approach for explosive percolation, Phys. Rev. E 81 (2010) 040101.

[48] D. Sankoff, C.-f. Zheng, A. Munoz, Z. y. Yang, Z. Adam, R. Warren, V. Choi , Q. Zhu, Issues in the Reconstruction of Gene Order Evolution, J. Comp. Sci. Tech. 25 (2010) 10.

[49] Y. Kim, Y.-k. Yun, S.-H. Yook, Explosive percolation in a nanotube-based system, Phys. Rev. E 82 (2010) 061105.

[50] D. Foster, J. Foster, M. Paczuski, P. Grassberger, Communities, clustering phase transitions, and hysteresis: Pitfalls in constructing network ensembles, Phys. Rev. E 81 (2010) 046115.

[51] Y. S. Cho, B. Kahng, D. Kim, Cluster aggregation model for discontinuous percolation transitions, Phys. Rev. E 81 (2010) 030103. 
[52] F. Radicchi, S. Fortunato, Explosive percolation: A numerical analysis, Phys. Rev. E 81 (2010) 036110.

[53] J. S. Andrade Jr. H. J. Herrmann, A. A. Moreira, C. L. N. Oliveira, Transport on exploding percolation clusters, Phys. Rev. E 83 (2011) 031133.

[54] J. Nagler, A. Levina, M. Timme, Impact of single links in competitive percolation, Nat. Phys. 7 (2011) 265 .

[55] Y. S. Cho, B. Kahng, Suppression Effect on Explosive Percolation, Phys. Rev. Lett. 107 (2011) 275703.

[56] R. A. da Costa, S. N. Dorogovtsev, A. V. Goltsev, J. F. F. Mendes, Explosive Percolation Transition is Actually Continuous, Phys. Rev. Lett. 105 (2010) 255701.

[57] O. Riordan, L. Warnke, Explosive Percolation Is Continuous, Science 333 (2011) 322.

[58] H. K. Lee, B. J. Kim, H. Park, Continuity of the explosive percolation transition Phys. Rev. E 84 (2011) 020101(R).

[59] P. Grassberger, C. Christensen, G. Bizhani, S. W. Son, M. Paczuski, Explosive Percolation is Continuous, but with Unusual Finite Size Behavior, Phys. Rev. Lett. 106 (2011) 225701.

[60] K. Christensen, N. R. Moloney, Complexity and Criticality, (Fudan University Press, Shanghai, 2006).

[61] H. Watanabe, C.-K. Hu, Mapping functions and critical behavior of percolation on rectangular domains, Phys. Rev. E 78 (2008) 041131.

[62] C.-P. Zhu, L.-T. Jia, B. J. Kim, B.-H. Wang, H. E. Stanley, Critical Points of Correlated Percolation in a Gravitational Link-adding Network Model, arXiv:1204.1482v1.

[63] C.-K. Hu, Percolation, clusters, and phase transitions in spin models, Phys. Rev. B 29 (1984) 5103-5108.

[64] C.-K. Hu, C.-Y. Lin, J.-A. Chen, Universal scaling functions for site and bond percolations on planar lattices, Physica A 221 (1995) 80-88.

[65] Chin-Kun Hu and N. Sh. Izmailian, Exact correlation functions of Bethe lattice spin models in external magnetic fields Phys. Rev. E 58 (1998) 1644.

[66] F. Y. Wu, The Potts model, Rev. Mod. Phys. 54 (1982) 235-266.

[67] C.-N. Chen, C.-K. Hu, F. Y. Wu Partition Function Zeros of the Square Lattice Potts Model Phys. Rev. Lett. 76 (1996) 169-172.

[68] F. Y. Wu, G. Rollet, H. Y. Huang, J. M. Maillard, Chin-Kun Hu, and Chi-Ning Chen, Directed 
Compact Lattice Animals, Restricted Partitions of an Integer, and the Infinite-State Potts Model, Phys. Rev. Lett. 76, 173 State Potts Model, Phys. Rev. Lett. 76 (1996) 173.

[69] F.-G. Wang, C.-K. Hu, Universality in dynamic critical phenomena, Phys. Rev. E 56 (1997) 2310 .

[70] Ming-Chya Wu, Chin-Kun Hu, and N. Sh. Izmailian, Universal finite-size scaling functions with exact nonuniversal metric factors Phys. Rev. E 67 (2003) 065103(R).

[71] C.-K. Hu, Site-bond-correlated percolationand a sublattice-dilute Potts model at finite temperatures, Phys. Rev. B 29 (1984) 5109-5116.

[72] R. H. Swendsen, J.-S. Wang, Nonuniversal critical dynamics in Monte Carlo simulations, Phys. Rev. Lett. 58 (1987) 86-89.

[73] C.-K. Hu, K.-S. Mak, Monte Carlo study of the Potts model on the square and the simple cubic lattices Phys. Rev. B 40 (1989) 5007-5014.

[74] L.-J. Chen, C.-K. Hu, K.-S. Mak, FORTRAN code for the cluster Monte Carlo study of the q-state Potts model on D-dimensional hypercubic lattices, Computer Physics Communications 66 (1991) 377-382. 
Table I: Critical points $T_{c}$ and critical exponents $\beta_{1}$ of $d$-dependent percolation probability $C_{1}$ for a site to be in the percolating cluster with strategy $S_{\max }$ and Case 1

\begin{tabular}{llllllllll}
\hline \hline$d$ & 0.01 & 0.1 & 0.2 & 0.5 & 0.8 & 1.0 & 1.2 & 1.5 & 2.0 \\
\hline$T_{c}$ & $0.356(5)$ & $0.358(3)$ & $0.369(3)$ & $0.408(3)$ & $0.442(7)$ & $0.460(6)$ & $0.474(9)$ & $0.491(0)$ & $0.509(0)$ \\
\hline$\beta_{1}$ & $0.894(8)$ & $0.943(7)$ & $0.971(1)$ & $0.984(9)$ & $1.01(1)$ & $0.990(3)$ & $1.00(6)$ & $1.02(7)$ & $1.02(6)$ \\
\hline \hline
\end{tabular}

Table II: Critical points $T_{c}$ and critical exponents $\beta_{2} d$-dependent percolation probability $C_{2}$ for a site to be in the percolating cluster with strategy $S_{\min }$ and Case $2, R=5$.

\begin{tabular}{|c|c|c|c|c|c|c|}
\hline $\begin{array}{ll}d & 0.2\end{array}$ & 0.5 & 0.8 & 1.0 & 1.2 & 1.5 & 2.0 \\
\hline$T_{c} 0.874(8)$ & $0.863(7)$ & $0.846(6)$ & $0.831(7)$ & $0.816(8)$ & $0.792(0)$ & $0.742(6)$ \\
\hline$\beta_{2} 1.18(2)$ & $1.34(4)$ & $1.28(0)$ & $1.31(9)$ & $1.26(0)$ & $1.25(0)$ & $1.26(1)$ \\
\hline
\end{tabular}



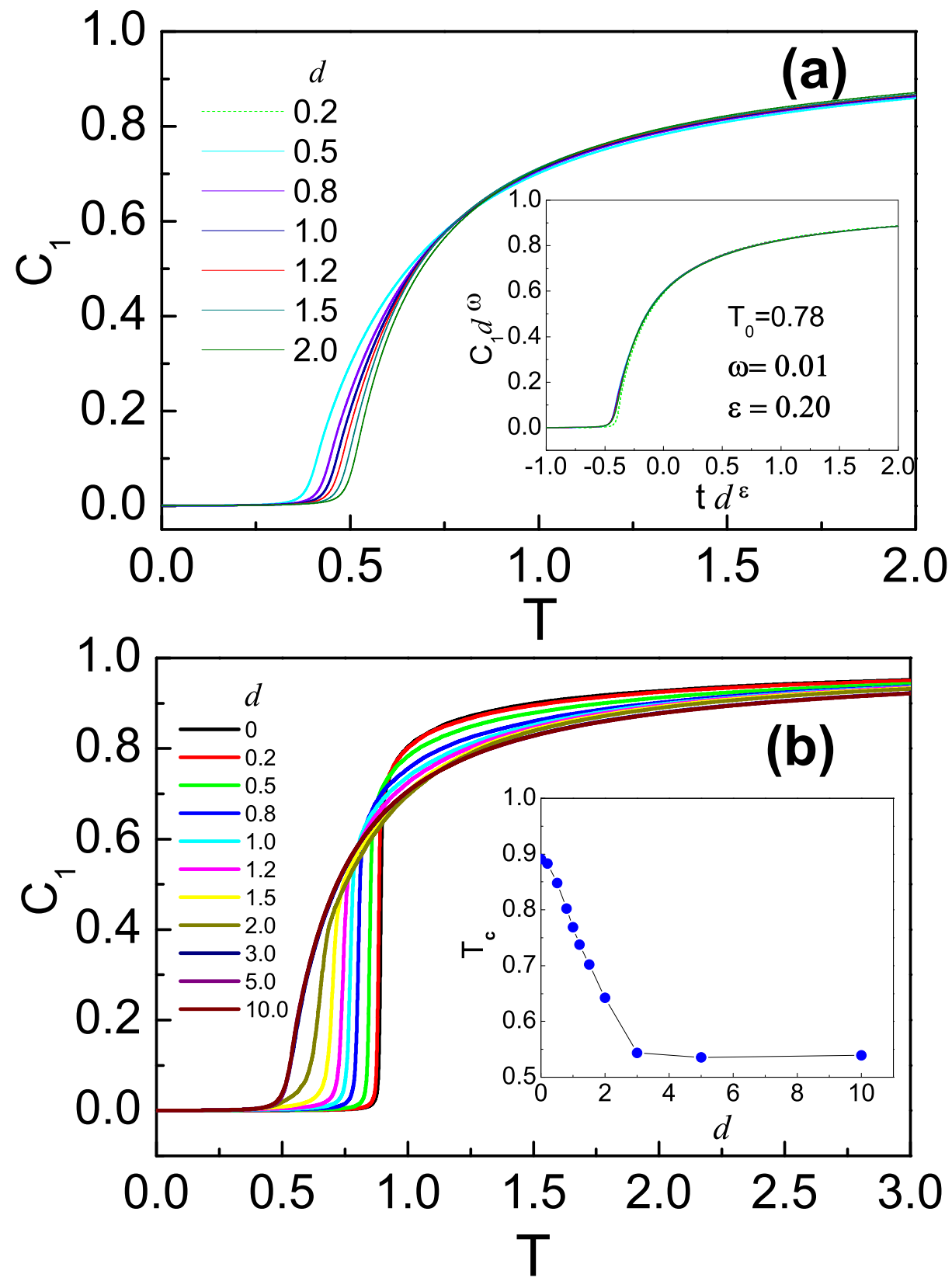

Figure 1: (color online) Node fraction $C_{1}(T)$ of the largest component in Case $1(R \rightarrow \infty)$. (a) For strategy $S_{\max }$. Inset: Re-scaled $C_{1} d^{\omega}$ as a function of $t d^{\epsilon}$ with $T_{0}=0.78$ and $t=\left(T-T_{0}\right) / T_{0}$. (b) $C_{1}(T)$ for strategy $S_{\text {min }}$. Inset: $T_{c}$ vs. $d$. For both (a) and (b) cases, $L=128$. 


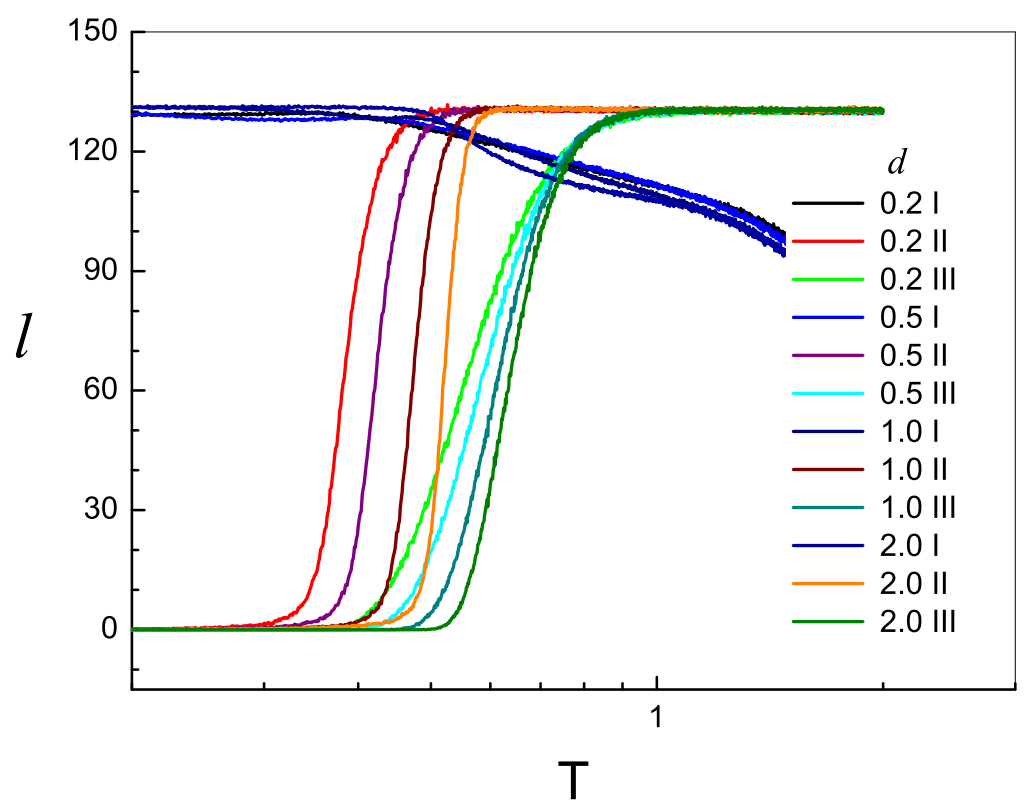

Figure 2: (color online) The average lengths $l$ with strategy $S_{\max }$ versus time steps $T$ in Case 1 $(R \rightarrow \infty)$ for different exponents $d=0.2,0.5,1.0$, and 2.0. In all cases, $L=128$. The finally saturated average lengths of both type-II and type-III links are $l_{0}=131.9$. 


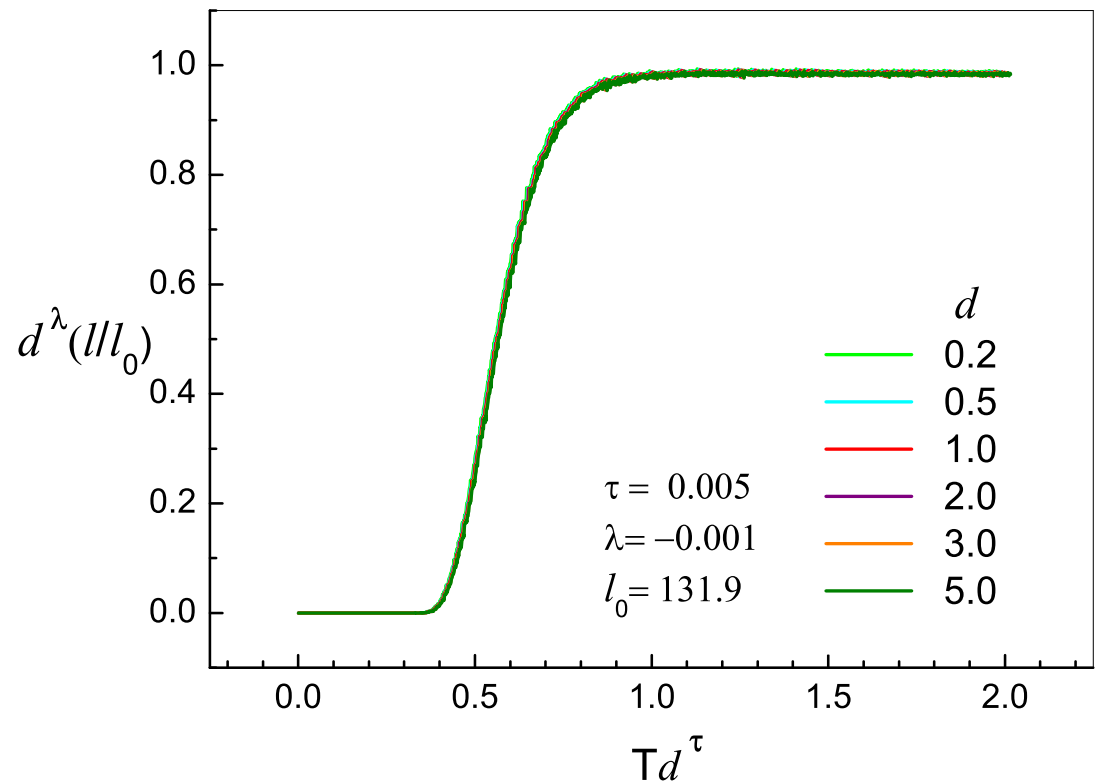

Figure 3: (color online) Rescaling normalized average lengths $d^{\lambda}\left(l / l_{0}\right)$ as a function of $T d^{\tau}$ for type-III links with $l_{0}=131.9$ (see Fig.2), $\lambda=-0.001$, and $\tau=0.005$. For all cases, $L=128$. 


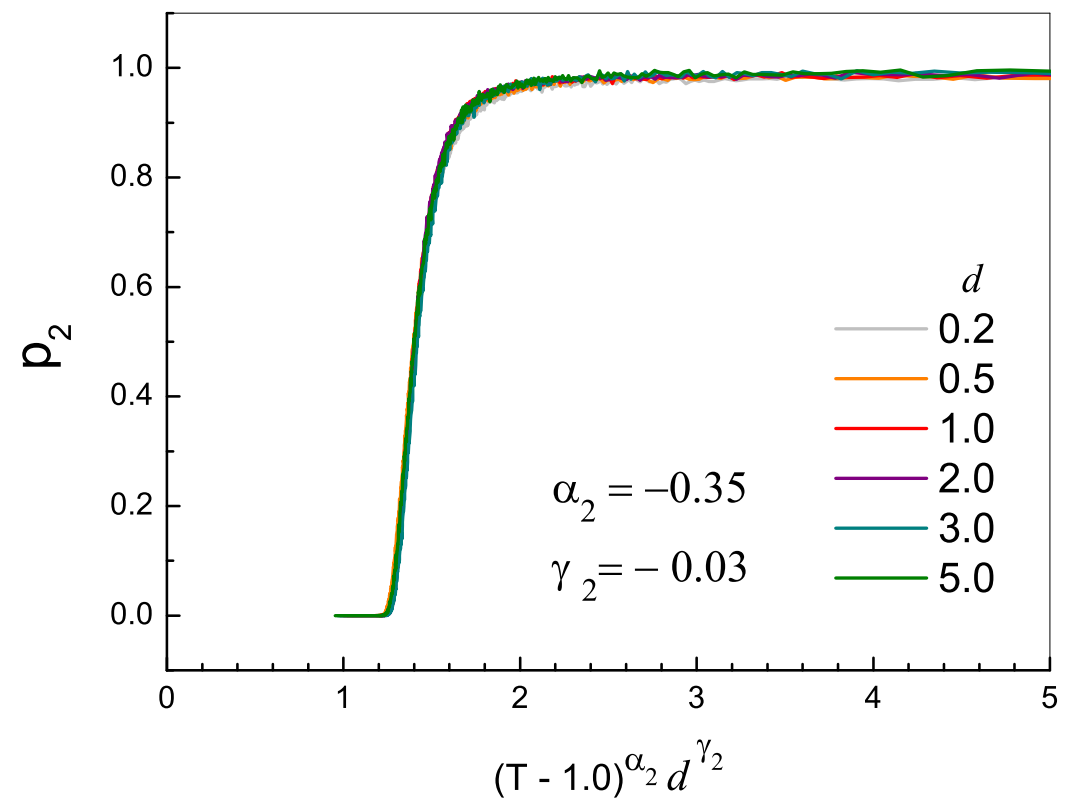

Figure 4: (color online) The probability for type-II links to arrive at saturated average length $l_{0}$ is represented with universal function $P_{2}$ which is obtained by rescaling normalized $l_{I I}$ of them (see Fig.2) with $d=0.2,0.5,1.0,2.0,3.0$ and 5.0, respectively. $L=128$. 


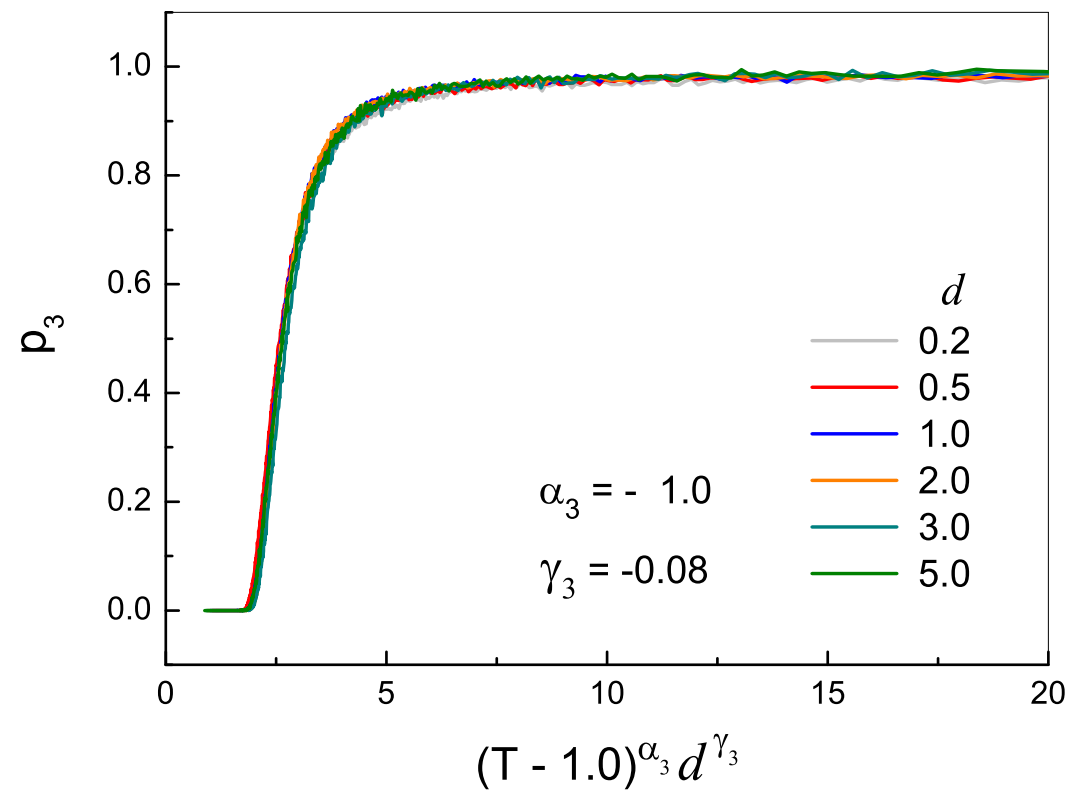

Figure 5: (color online) The probability for type-III links to arrive at saturated average length $l_{0}$ is represented with universal function $P_{3}$ which is obtained by rescaling normalized $l_{I I I}$ of them (see Fig.2) with $d=0.2,0.5,1.0,2.0$ and 3.0, respectively. $L=128$. 


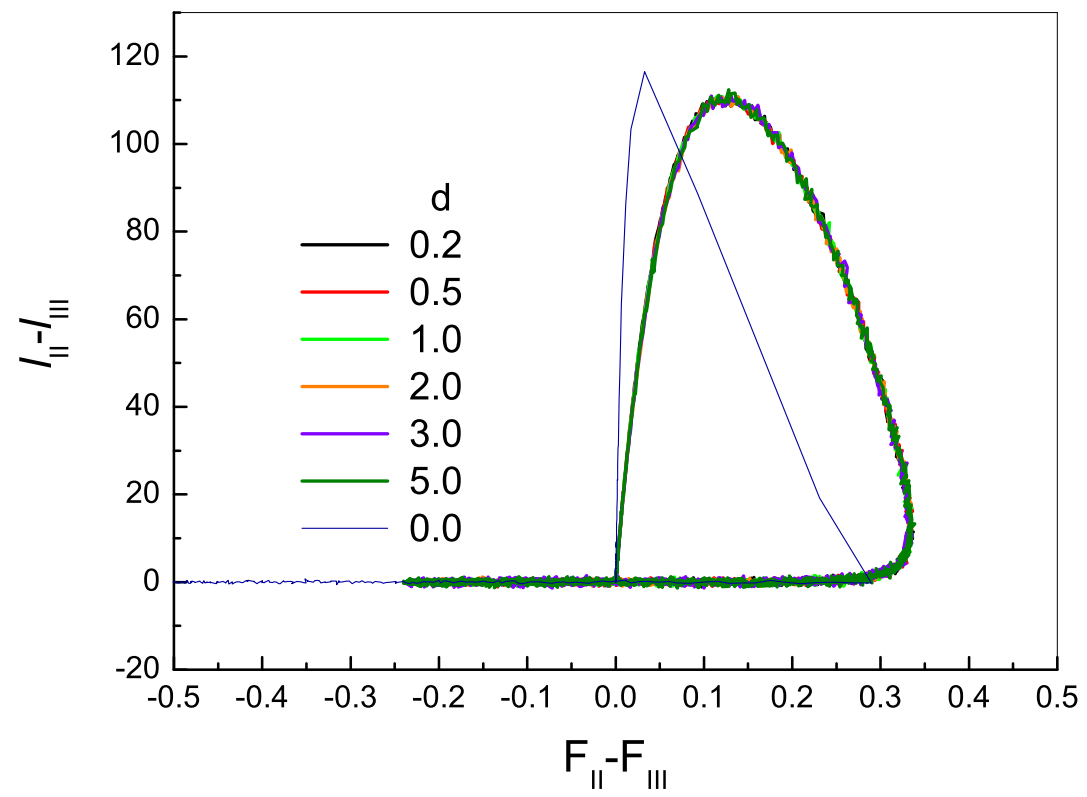

Figure 6: (color online) The differences of average lengths between type-II and type-III links versus that of average fractions between these two types of links with $d=0.2,0.5,1.0,2.0,3.0$ and 5.0 at the same $T$, respectively. $L=128$. 

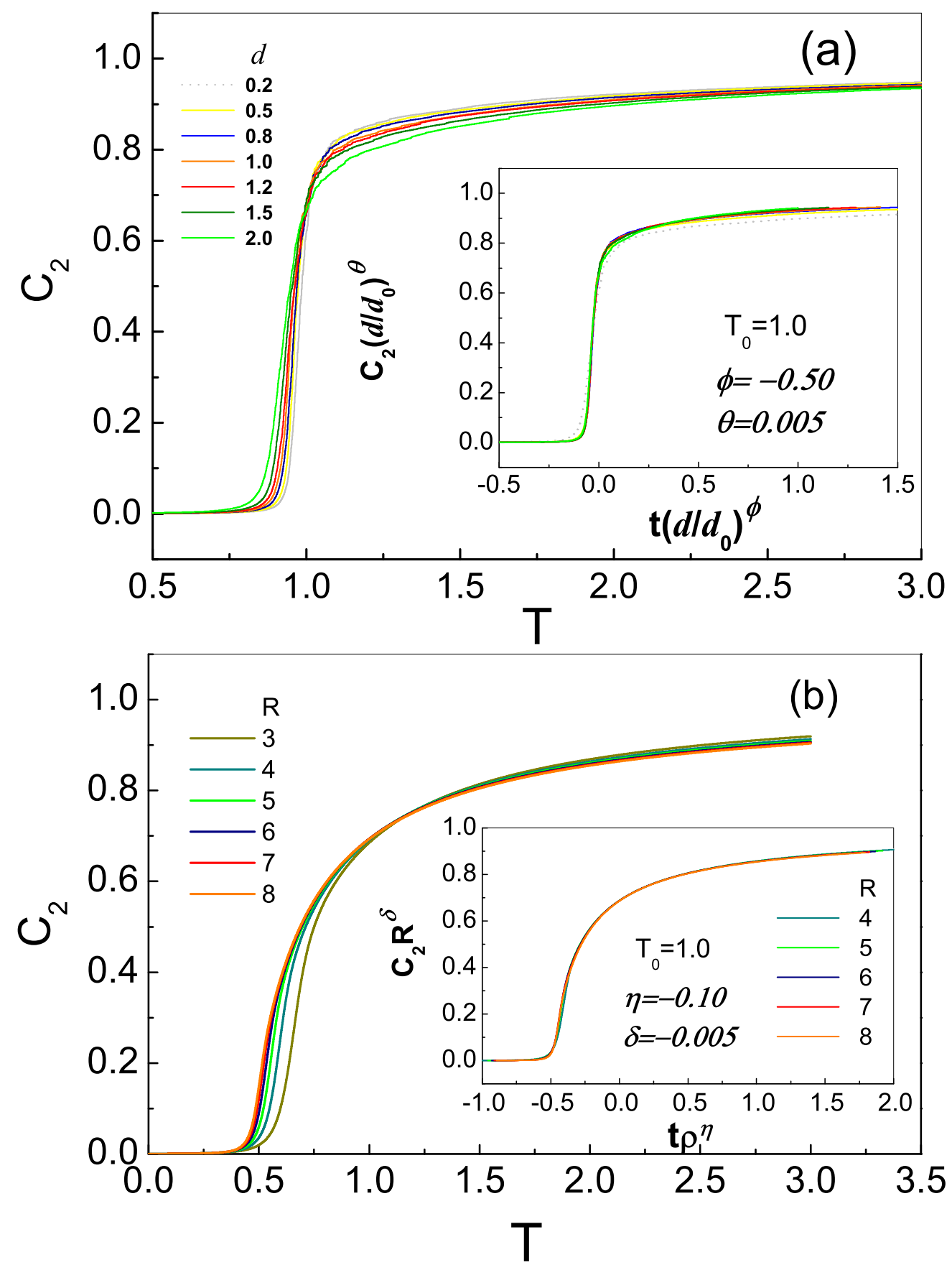

Figure 7: (color online) Node fraction $C_{2}(T)$ of the largest component in Case 2. $(R<\infty$ and $d>0$ ). (a) $S_{\min }$ with $R=5$. Inset: Re-scaled $C_{2}(T)$ with $T_{0}=1.0$. (b) $S_{\max }$ with $d=2.0$. Inset: Re-scaled $C_{2}(T)$ with $R_{0}=2 . L=128$ for all cases. 

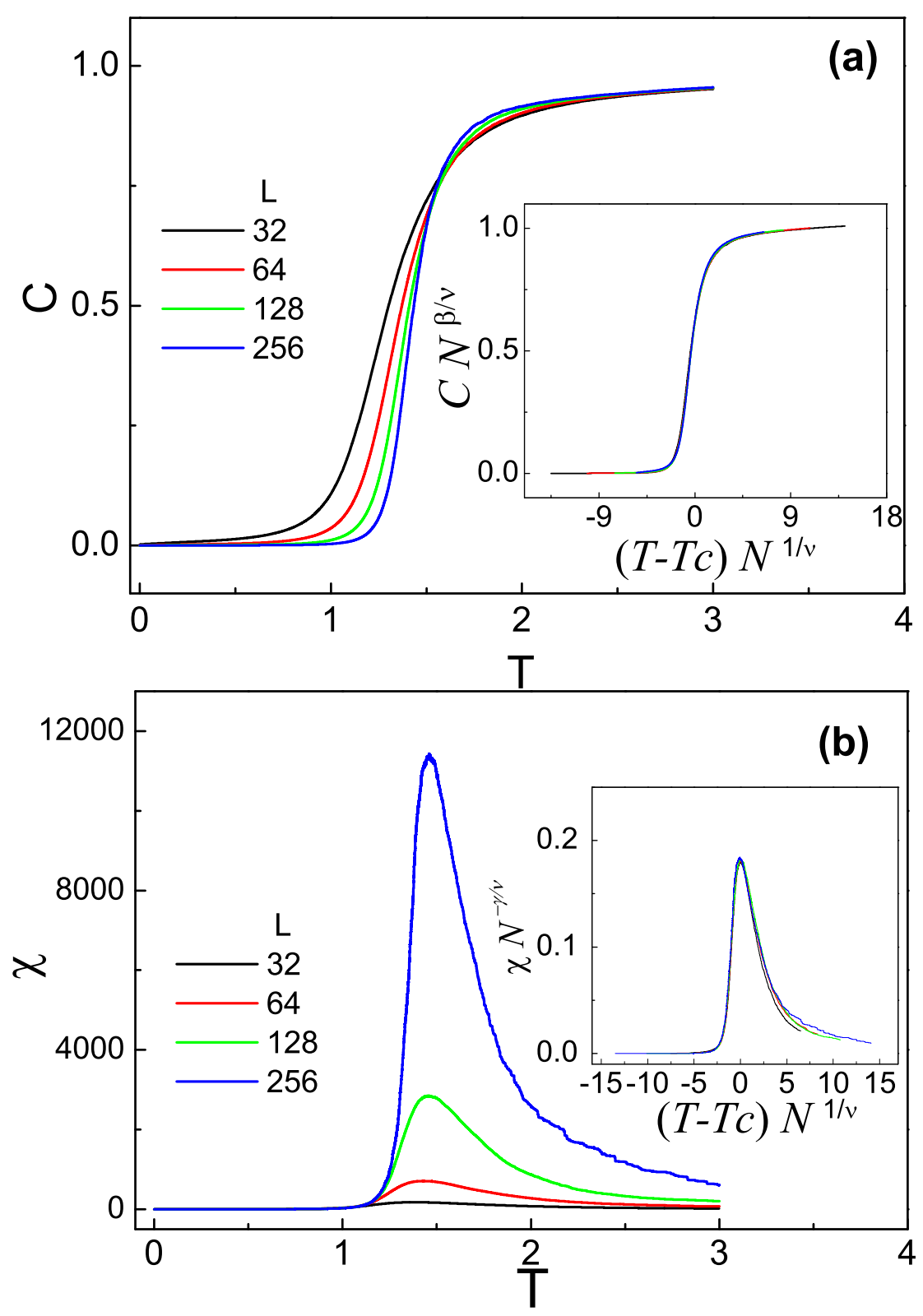

Figure 8: (color online)(a) $C_{2}(T)$ for $S_{\min }$ and (Inset) its finite-size scaling. (b) $\chi(T)$ for $S_{\min }$ and (Inset) its finite-size scaling. With $L=32,64,128$ and $256 ; R=2, d=2.0$ for both panels. 